\title{
L'enseignement musical universitaire et son prolongement en IUFM
}

Pierre Gaucher

\section{(2) OpenEdition}

1 Journals

Édition électronique

URL : http://journals.openedition.org/trema/368

DOI : $10.4000 /$ trema.368

ISSN : 2107-0997

Éditeur

Faculté d'Éducation de l'université de Montpellier

\section{Édition imprimée}

Date de publication : 1 décembre 2005

ISSN : 1167-315X

\section{Référence électronique}

Pierre Gaucher, «L'enseignement musical universitaire et son prolongement en IUFM », Tréma [En ligne], 25 | 2005, mis en ligne le 11 janvier 2010, consulté le 20 avril 2019. URL : http://

journals.openedition.org/trema/368; DOI : 10.4000/trema.368

Ce document a été généré automatiquement le 20 avril 2019.

Trema 


\title{
L'enseignement musical universitaire et son prolongement en IUFM
}

\author{
Pierre Gaucher
}

1 Les premières Universités françaises apparurent au cours du treizième siècle : Paris (1215), Montpellier (1220), Toulouse (1229)... La musique y figurait dans le cadre de la faculté des arts. L'enseignement comprenait deux parties, le trivium

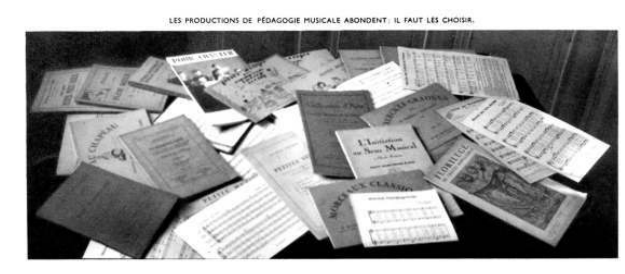
(grammaire, rhétorique, dialectique) et le quadrivium (astronomie, arithmétique et géométrie, musique). Ce cursus permettait d'accéder aux facultés supérieures de droit, de médecine ou de théologie.

2 Toutefois, la musique perdit peu à peu ses lettres de noblesse avant de disparaître complètement à la Renaissance. Elle fut totalement négligée lorsque la Convention supprima le 15 septembre 1793 les vingt-et-une Universités héritées de l'Ancien régime'.

3 Dans un contexte universitaire complexe et ramifié, l'enseignement supérieur de la musique apparait comme une discipline relativement récente et représente depuis peu de temps une spécialité à part entière. Même si, pendant longtemps, l'enseignement de l'histoire de la musique notamment fut l'apanage des Conservatoires, Michel DELAHAYE et Danièle PISTONE expliquent que la musicologie refit progressivement surface en Université par l'intermédiaire de conférences en 1895 à la Sorbonne ${ }^{2}$, puis au Collège de France et à l'Ecole des Hautes Etudes ${ }^{3}$. Plusieurs initiatives contemporaines sont aussi à souligner en province (Strasbourg ${ }^{4}$, Lyon, Grenoble, Poitiers...). 
La Sorbonne concourut à elle seule à établir la légitimité de la musicologie en France. La musique y fut implantée par Romain ROLLAND (1866-1944), chargé de cours de l'histoire de l'art en 1904, suppléé ensuite par Louis LALOY, puis par André PIRRO (1869-1943) chargé de cours d'histoire de la musique. Paul-Marie MASSON (1882-1954), maître de conférences en 1933, obtint la création de l'Institut de Musicologie en 1951. Atteint par la limite d'âge, il fut remplacé par Jacques CHAILLEY un an plus tard.

Ces études furent assez longtemps confidentielles : 62 étudiants en $1962 ; 85$ en $1965^{5}$. Une évolution spectaculaire se fit jour en 1970 après la création de l'UER de musicologie qui mit en place un cursus jusqu'en Maîtrise :

\section{Evolution du nombre d' étudiants à la Sorbonne}

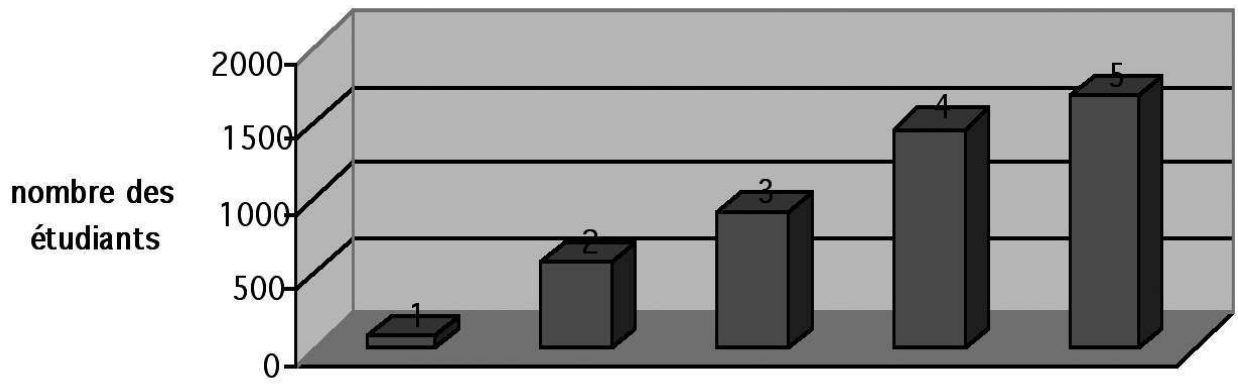

Outre la Sorbonne, la plupart des créations de départements de musicologie datent des années 1970. Au jour d'aujourd'hui, un peu moins du tiers des Universités françaises dispensent un enseignement musical ${ }^{6}$.

7 Tandis que le terme musicologue apparaît en 1889, la musicologie, science de la théorie, de l'esthétique et de l'histoire de la musique, se développe ainsi considérablement au vingtième siècle après avoir été longtemps oubliée. Etymologiquement voisin de celui de musicographie, à savoir celui qui écrit sur la musique, la musicologie recouvre un large ensemble d'activités techniques et historiques qui suppose des ramifications nombreuses. Armand MACHABEY précise qu' « il peut paraître facile de dresser l'inventaire des facteurs médiats et immédiats qui concourent à la création et à la divulgation d'une partition, il est déjà moins facile d'en faire l'historique et souvent malaisé de déterminer les causes profondes de cette création, ses incidences sur l'évolution musicale, ses attaches secrètes avec la société contemporaine. $»^{7}$

Le musicologue est ainsi solidaire et tributaire de nombreux champs de recherches. Outre un ensemble de matières techniques obligatoire lié à la spécificité de l'art musical (formation musicale, écriture notamment), le nombre de disciplines dans le cursus universitaire ne cesse de croître en rapport avec l'évolution des courants musicaux (ethnomusicologie, jazz, musiques actuelles...), mais aussi en liaison avec d'autres spécialités à dominante scientifique (acoustique, organologie, musicothérapie...). 
Dans ce contexte, il est important de souligner que l'éducation musicale n'est apparue à l'Université que plus récemment encore. Dans un premier temps, les cursus furent souvent restreints à la Licence, voire au seul DEUG. Dorénavant, un premier palier forme les étudiants en trois ans jusqu'en Licence, avant de leur proposer deux voies principales, l'enseignement ou la recherche (Master, puis Doctorat).

Michel DELAHAYE et Danielle PISTONE indiquent le niveau requis pour prétendre réussir ses études en musicologie :

- "un bon niveau de solfège (clef de sol et de fa 4e ligne) et de dictée musicale à une ou deux voix;

- l'acquis solide de la théorie musicale;

- la maîtrise d'un instrument polyphonique (piano de préférence);

- de bonnes aptitudes au chant ;

- un réel entraînement au déchiffrage (vocal et instrumental), compte tenu du grand nombre de textes musicaux que l'étudiant aura à approcher durant ces années pour compléter sa formation historique et technique;

- une bonne culture générale.

- Il faudra s'efforcer aussi d'acquérir l'audition interne, c'est-à-dire la faculté de juger immédiatement du résultat sonore de la partition lue. Quant à l'écriture musicale (harmonie surtout), l'acoustique, l'histoire de la musique, l'organologie, l'histoire de l'art, elles pourront en revanche être commencées lors de la première année universitaire. ${ }^{8}$

11 Et de ponctuer en soulignant que "l'ampleur des programmes et la diversité des matières enseignées rendent souvent salutaires, là encore, quelques approches personnelles préalables... ${ }^{9}$. En somme, les études universitaires impliquent au moins une bivalence technique et historique qui se retrouve dans l'intitulé complet des départements de formation: musique-musicologie.

Les maquettes de formation ont été profondément renouvelées cette année dans le cadre de la réforme $\mathrm{LMD}^{10}$, diplômes communs à tous les pays de l'espace européen. A chaque niveau correspond un nombre d'ECTS (European Credit Transfer System) calculés selon différents facteurs tels que le volume horaire des cours, le travail personnel ou encore les stages. Les études sont organisées en semestres et non plus en années. Chaque semestre nécessite la validation de trente ECTS.

La Revue de musicologie a publié en 2001 un annuaire national des établissements d'enseignement supérieur et de recherche ${ }^{11}$. Il apparaît que les établissements les plus récents sont souvent ceux qui proposent le moins d'offres de formation (Grenoble,1974; Besançon, 1976; Saint-Etienne, 1980). Cinq départements préparent en plus au CAPES (Bordeaux, Dijon, Rouen, Nice, Poitiers). Treize autres enfin présentent un cursus complet comprenant aussi l'agrégation : Aix-Marseille, Lille, Lyon, Metz, Montpellier, Nancy, Paris IV (Sorbonne), Paris VIII, Reims, Rennes, Strasbourg, Toulouse et Tours. 
Le Certificat d'aptitude professionnel à l'enseignement du second degré (CAPES) fut créé en 1950. Il fallut néanmoins attendre 1972-1973 pour que soit institué le concours en éducation musicale et chant choral. Ce concours succéda au CAEM (Certificat d'aptitude à l'enseignement musical, premier et second degrés) auquel prépara le Lycée La Fontaine à Paris de 1947 à 1974.

sa part, l'agrégation externe fut créée en 1974-1975. Elle ne comporte pas d'épreuve sur dossier et constitue ainsi le seul concours qui échappe complètement à l'IUFM. Depuis $2000^{12}$, se démarquant en cela des autres concours de recrutement, le diplôme est une agrégation de "musique" et non plus d' "éducation musicale et chant choral", intitulé qui tendait à sceller la perspective professionnelle dans le second degré, quand bien même les perspectives d'emploi dans le supérieur demeurent toujours assez rares.

Rappelons que l'Université eut longtemps pour responsabilité de former seule l'ensemble des enseignants du secondaire. Cette mission incombe dorénavant aux IUFM qui conservent dans la pratique un partenariat avec les départements de musicologie locaux, notamment pour la préparation des épreuves d'admissibilité du CAPES et de l'agrégation interne :

"Notons qu'en moins de trente ans [...] un effort considérable de recrutement et de formation a permis de combler un déficit quantitatif et qualitatif très important et qu'aujourd'hui la compétence des professeurs d'éducation musicale et de chant choral est volontiers plébiscitée par les chefs d'établissement. ${ }^{13}$

1990 initia une réforme de la formation des enseignants des premier et second degrés aboutissant à la création des IUFM (Instituts Universitaires de Formation des Maîtres) en remplacement des Ecoles normales. L'ouverture de nombreux IUFM eut lieu en septembre $1991^{14}$. épreuves d'admissibilité et quatre épreuves d'admission dont la musique (au choix entre langues vivantes et arts plastiques) ${ }^{15}$. Une première partie consiste en l'interprétation vocale ou instrumentale d'une œuvre choisie par le candidat ponctuée par un bref échange avec le jury. Il s'agit ensuite de soumettre un répertoire personnel de cinq chants adaptés aux élèves de l'école primaire. L'un d'entre eux est interprété de mémoire et $a$ capella. Enfin, le candidat analyse plusieurs œuvres musicales mises en rapport avec des pratiques pédagogiques ${ }^{16}$. De nouvelles épreuves sont annoncées pour la rentrée 2005-2006 ${ }^{17}$, évaluées durant un entretien avec le jury portant à la fois sur un dossier professionnel (système éducatif, métier, programmes...) et sur un exposé ou une performance dans les domaines de la musique, des arts visuels ou de la littérature de jeunesse. 

le Rectorat.
La seconde année de formation complète la formation initiale en s'adressant à l'ensemble des stagiaires. A charge pour chaque IUFM d'organiser les modalités de travail et de définir les volumes horaires. Dans les faits, la musique dans l'enseignement primaire est sans doute l'une des disciplines les plus négligées. Le Plan des arts et de la culture du 14 décembre 2000 rapportait que $72 \%$ des enseignants du premier degré reconnaissaient leur incompétence à enseigner la musique ${ }^{18}$.

Un constat moins pessimiste était tout aussi alarmant :

"Si l'on étudie les résultats d'une enquête menée en 1998 sur la polyvalence auprès de professeurs sortant de l'IUFM, la première discipline la plus difficile à enseigner serait l'éducation musicale pour $53 \%$, les sciences et techniques viennent ensuite avec $48 \%$ [...], On y apprend aussi que $50 \%$ d'enseignants n'enseignent pas la musique seuls. ${ }^{19}$

La mise en place de dominantes en seconde année d'IUFM tend à pallier ces lacunes par un complément de formation et une ouverture sur les compétences des partenaires locaux. Il n'en demeure pas moins que la politique volontariste dans un premier temps, malheureusement plus velléitaire ces dernières années, confère à l'éducation musicale à l'école un caractère plus ou moins conjectural et aléatoire.

Pour le second degré, la première année est consacrée à la préparation au concours national dispensée en partenariat avec une Université de rattachement. Le programme consiste en une remise à niveau dans tous les domaines disciplinaires et une préparation aux épreuves écrites et orales. Chaque centre de formation procède aussi à ses propres aménagements $^{20}$. Le plus souvent, en concertation avec l'IUFM où sont inscrits les étudiants, l'Université prend en charge la préparation des épreuves écrites, tandis que l'oral dépend plus généralement des IUFM à qui incombe en particulier l'épreuve sur dossier. Enfin, l'organisation des stages en établissement s'effectue en concertation avec

Les textes du concours du CAPES externe ont été modifiés en $2001^{21}$. Ils accordent une plus large part à l'oral par le jeu des coefficients. Un ensemble d'épreuves techniques et une dissertation composent l'admissibilité (coefficient 2). Les épreuves d'admission confèrent à la musicalité des candidats une place essentielle: arrangement éventuellement informatisé (coefficient 1), direction de chœur (coefficient 1) et épreuve sur dossier (coefficient 2) requièrent l'ensemble des compétences requises pour l'enseignement de la musique. 
Dans le même temps, le nombre de postes ne cesse de décroître de manière inquiétante pour la discipline :

\section{Nombre de postes (CAPES externe)}

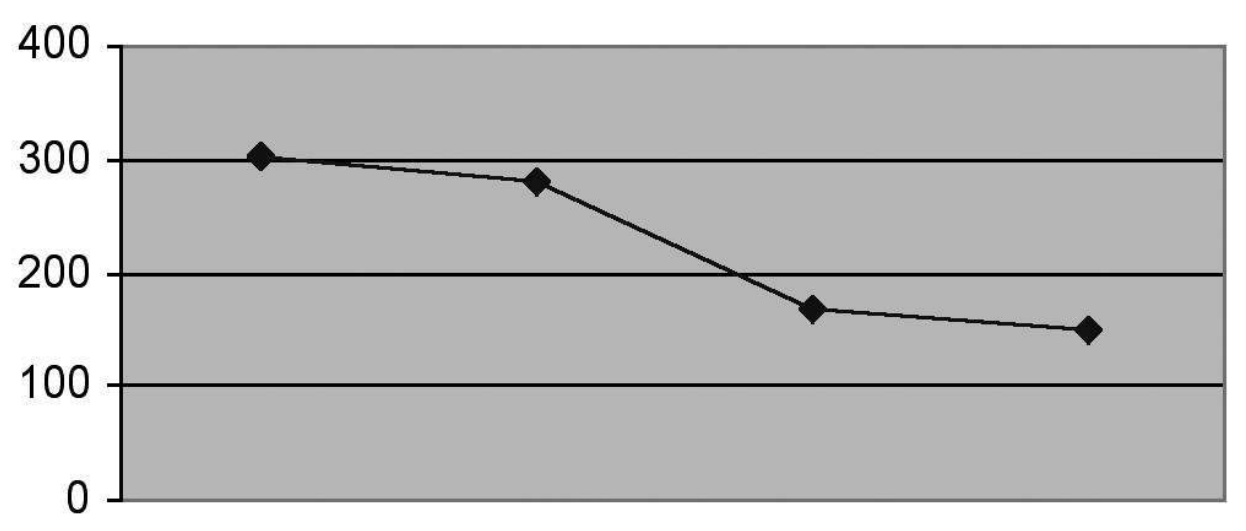

Le CAPES externe est de loin le concours qui engage le plus grand nombre de professeurs. La réduction des postes ( $40 \%$ l'an passé et $12 \%$ cette année) représente, au final et pour l'année en cours, un total de postes deux fois moindre qu'il y a seulement trois ans. Les autres concours sont aussi touchés, tel le CAFEP (Certificat d'aptitude aux fonctions d'enseignement dans les établissements d'enseignement privé du second degré sous contrat) formation proposée en IUFM, ainsi qu'à l'Université catholique de l'Ouest (Angers) structurée dernièrement ${ }^{22}$. Le CAFEP comporte les mêmes épreuves que le CAPES externe mais procède d'un classement différent.

La seconde année en IUFM confirme le plus souvent la réussite aux concours par une formation professionnelle ou pratique en alternance. La formation se déroule essentiellement sur deux lieux :

- lieu d'exercice du stage en responsabilité (4 à 6 heures de cours hebdomadaires en pleine responsabilité auxquelles s'ajoutent éventuellement le suivi d'une chorale).

- l'IUFM pour les formations transversales et disciplinaires (deux jours de formation hebdomadaires). Pour la formation transversale, les lauréats du CAFEP sont réunis par les ARPEC (Associations régionales pour la promotion pédagogique et professionnelle de l'enseignement catholique), partenaires des IUFM. Un stage de pratique accompagnée dans un collège autre que celui d'affectation et un mémoire professionnel complètent l'année.

31 La formation fait l'objet d'une appréciation de la part de l'IUFM, conclusions transmises au Rectorat et examinées en jury académique pour titularisation des professeurs stagiaires. Dans le cas d'une non-validation, une seconde année est généralement accordée aux stagiaires afin de parfaire leur pratique professionnelle.

Seize IUFM sur un total d'une trentaine proposent une formation musicale aux lauréats des concours externes du CAPES, du CAFEP et de l'agrégation: Aix-Marseille, Bordeaux, 
Créteil, Dijon, Lille, Lyon, Montpellier, Nancy-Metz, Orléans-Tours, Paris, Poitiers, Reims, Rennes, Rouen, Strasbourg, Toulouse.

Outre la formation initiale, l'IUFM a aussi pour mission de former les enseignants tout au long de leurs carrières depuis la suppression des Missions académiques à la formation des personnels de l'Education nationale (MAFPEN) en 1998. Les Inspections académiques et les Rectorats ont progressivement repris la main depuis en définissant les plans de formation.

Pour l'enseignement primaire, l'IUFM assure la formation au Certificat d'aptitude aux fonctions d'instituteur ou de professeur des écoles maitre formateur (CAFIPEMF). Ce diplôme se décline en deux catégories : avec ou sans option de spécialisation qui est fonction de l'option choisie par le candidat, au rang desquelles figure l'éducation musicale ${ }^{23}$. Les candidats, instituteurs ou professeurs des écoles, doivent
années de services effectifs d'enseignement à temps complet.

Les titulaires du CAFIPEMF sans option ont vocation à exercer en tant que conseillers pédagogiques de circonscription généralistes ou en tant que maîtres formateurs. Les enseignants titulaires d'un CAFIPEMF spécialisé peuvent postuler sur des postes de conseillers pédagogiques départementaux ou de maîtres formateurs.

Le certificat d'aptitude aux fonctions d'instituteur ou de professeur des écoles maître formateur comprend une épreuve d'admissibilité et deux épreuves d'admission ${ }^{24}$. Outre la préparation critique de séances de cours, l'IUFM encadre aussi le mémoire rédigé par le candidat et portant sur l'un des domaines au programme de l'école primaire ou du collège pour les SEGPA ${ }^{25}$. L'admissibilité a pour objet d'évaluer la compétence professionnelle des candidats. Les épreuves d'admission doivent permettre de déceler ceux qui peuvent allier à leurs qualités d'enseignant des qualités de formateur d'adultes et d'animateur pédagogique.

Pour ce qui concerne le secondaire, et bien que les chiffres soient en perpétuelle évolution, la France compte un peu moins de 400000 enseignants dont $1,5 \%$ de professeurs d'éducation musicale et chant choral ${ }^{26}$ répartis dans près de cinq mille établissements, essentiellement en collège ${ }^{27}$. Une très grande majorité des enseignants a suivi un parcours universitaire jusqu'en licence, niveau habituellement requis pour prétendre éventuellement à un poste de vacataire, avant de passer un CAPES, puis une agrégation. 
Différents IUFM proposent des préparations au CAPES et à l'agrégation internes ${ }^{28}$, creés respectivement en 1987-1988 et en 1989-1990. La nature de leurs épreuves fut modifiée lors de la session $2001^{29}$. Ces concours voient aussi le nombre de postes réduits du tiers depuis moins de dix ans. Ce qui fera un nombre total de recrutements ou de promotions d'à peine plus de deux cents sur le plan national pour l'année en cours :

\begin{tabular}{|l|l|}
\hline CAPES externe & 150 postes \\
\hline \hline CAPES interne & 20 postes \\
\hline agrégation externe & 28 postes \\
\hline agrégation interne & 15 postes \\
\hline
\end{tabular}

Cela n'est pas sans poser un sérieux problème en terme de perspectives d'emploi, alors que « les concours du CAPES et de l'agrégation constituent le débouché professionnel de la majorité des étudiants. $\|^{30}$

41 Quand bien même les taux de réussite aux concours en éducation musicale demeurent encore parmi les plus élevés toutes disciplines confondues ${ }^{31}$.

Dans le même temps furent institués des concours accessibles aux enseignants non titulaires. La création du CAPES réservé entre dans ce registre ${ }^{32}$. Le nombre des épreuves est considérablement allégé ${ }^{33}$ et celles-ci font essentiellement appel à l'expérience pédagogique acquise. Ce type de recrutement est toutefois assez minime, seuls huit postes étant ouverts lors de la session 2005.

Dernière forme de recrutement: l'examen professionnel, prévu pour une durée de cinq ans, est destiné aux personnels non titulaires en fonction, en vue de leur recrutement dans les corps d'enseignement, d'éducation ou d'orientation ${ }^{34}$. Comme le CAFIPEMF, il s'agit d'un examen et non d'un concours; déclinaison, au niveau de chaque académie, du concours réservé. Les candidats obtenant la moyenne sont stagiaires l'année suivante, avant d'être titularisés.

44 Enfin, il exista provisoirement au cours des années 1990 et jusqu'en 1998 le CAPES "spécifique ", intermédiaire entre le CAPES interne et le CAPES réservé qui fut aussi destiné à contribuer à la résorption de l'emploi précaire.

Outre une préparation aux différents concours, une majorité de stages de formation continue impliquent les IUFM tant en termes de compétences que d'infrastructures. Les responsables de l'enseignement primaire et secondaire (Inspecteurs d'Académie Inspecteurs Pédagogiques Régionaux) proposent et supervisent les contenus de formation en fonction des attentes des enseignants et des priorités ministérielles. La baisse des moyens alloués a entraîné une réduction significative et inquiétante du nombre de stages proposés ces dernières années. 
Nous ne saurions suspendre notre propos sans évoquer la recherche en IUFM dont le projet vise à la fois à permettre à tout formateur de s'investir dans une équipe universitaire (laboratoires) ou bien de contribuer à un groupe de recherche - innovation (programmes finalisés à échéance annuelle pour accompagner les évolutions des plans de formation). Les statuts des personnels enseignants sont communs en IUFM et en Université. Les thèmes de recherche se rejoignent parfois, dans le domaine de l'histoire de la discipline par exemple comme en attestent les travaux de Michèle ALTEN, co-auteur de ce numéro de TREMA, ou bien encore ceux de Jean-Pierre MIALARET en sciences de l'éducation musicale et didactique de la musique ${ }^{35}$.

47 En guise de conclusion, il n'est pas inutile de rappeler que l'IUFM, et dans une moindre mesure l'Université, sont actuellement victimes de vives critiques, souvent relayées par les médias. Et pour cause : leurs missions nombreuses se trouvent au cœur même du système éducatif français, en terme de formation initiale et continue. Les qualités disciplinaires fondamentales doublées d'une formation pédagogique doivent offrir une formation théorique et pratique complète et complémentaire.

Les perspectives d'avenir sont cruciales. Un nombre considérable d'enseignants seront à former dans les dix prochaines années, toutes disciplines confondues. Au-delà des prérogatives des uns et des autres, il convient de répondre au mieux aux attentes des étudiants, des stagiaires et des professeurs, gage d'une capacité à accompagner l'évolution progressive des contenus et des pratiques afin de tenter de répondre au mieux aux attentes nouvelles des élèves. L'IUFM se trouve au carrefour de l'enseignement dispensé en Université et de la profession sous la responsabilité de l'Inspection académique (primaire) et du Rectorat (secondaire). A l'aube d'un rattachement institutionnel des IUFM au sein des Universités, seule une concertation intelligente permettra de garantir efficacement la pérennité et le renforcement des compétences des enseignants et leurs capacités de renouvellement

\section{ANNEXES}




\section{Annexes}

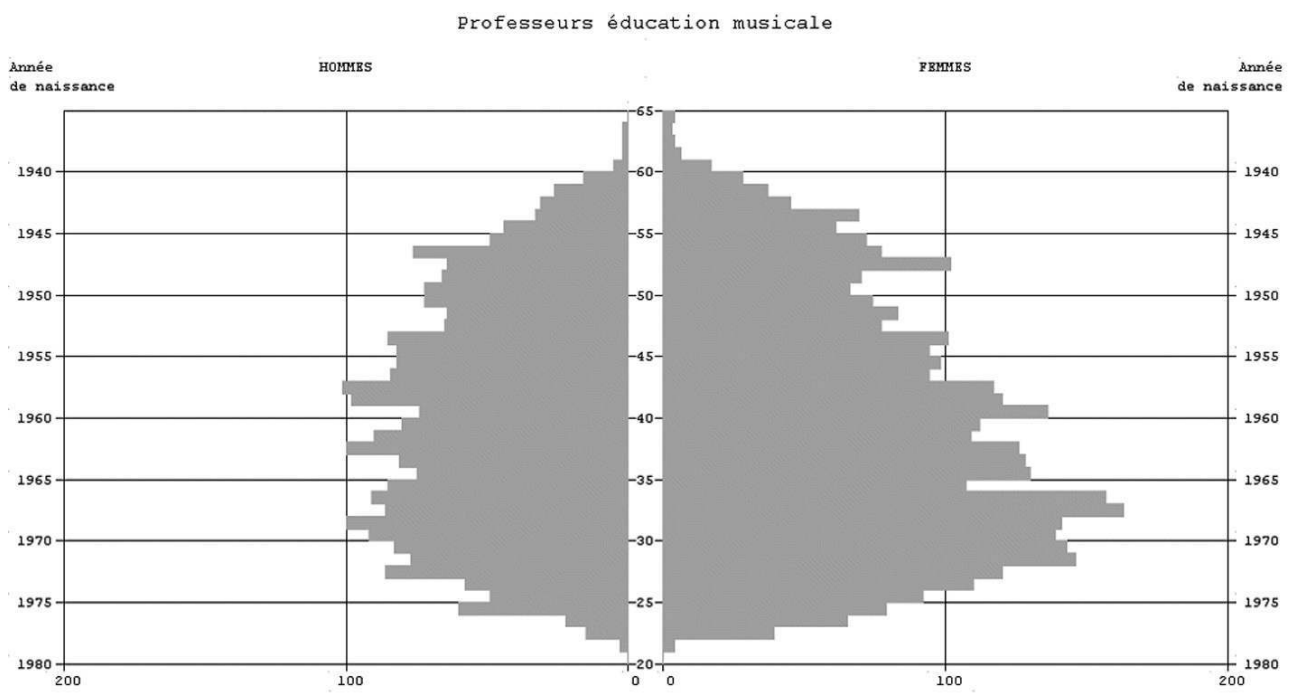

-.... pyxamides âg ș

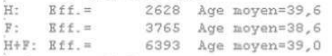

Annexe 1 : Pyramide des âges des professeurs d'éducation musicale

Ces données figurent sur l'excellent site Educnet : http ://www.educnet.education.fr/ musique/accueil/index.htm 


\begin{tabular}{|c|c|c|c|c|c|c|c|c|c|c|c|c|c|c|}
\hline Concours & Année & Postes & Inscrits & Présents & Pré/Poste & $\begin{array}{l}\text { \% Prés } \\
\text { Insc. }\end{array}$ & Admisesiblks & $\begin{array}{l}\% \text { admis } \\
\text { /Presents }\end{array}$ & Admis & $\begin{array}{l}\text { Admis / } \\
\text { Présents }\end{array}$ & $\begin{array}{l}\% \text { Admis } \\
\text { /Postes }\end{array}$ & LC & Total & $\begin{array}{c}\% \text { Postes } \\
\text { départ }\end{array}$ \\
\hline \multirow[t]{9}{*}{$\begin{array}{l}\text { Agents } \\
\text { externes }\end{array}$} & 1995 & $\begin{array}{c}59 \\
1996\end{array}$ & 59 & 122 & 134 & & & & 21 & 29 & & & & \\
\hline & 1997 & 59 & & 136 & & & & & 22 & & & & & \\
\hline & 1998 & 51 & 240 & 148 & 2,9 & 61,67 & 53 & 35,81 & 26 & 17,57 & 50,98 & & 26 & 50,98 \\
\hline & 1999 & 40 & 228 & 133 & 3,3 & 58,33 & 53 & 39,85 & 33 & 24,81 & 82,50 & & 33 & 82,50 \\
\hline & 2000 & 32 & 212 & 119 & 3,7 & 56,13 & 58 & 48,74 & 32 & 26,89 & 100 & & 32 & 100 \\
\hline & 2001 & 34 & 248 & 143 & 4,2 & 57,66 & 58 & 40,56 & 34 & 23,78 & 100 & & 34 & 100 \\
\hline & 2002 & 36 & 266 & 195 & 5,4 & 73,31 & 73 & 37,44 & 36 & 18,46 & 100 & & 36 & 100 \\
\hline & 2003 & 36 & 273 & 190 & 5 & 69,60 & 74 & 38,95 & 36 & 18,95 & 100 & & 36 & 100 \\
\hline & 2004 & 28 & 279 & 182 & 7 & 65,23 & 62 & 34,07 & 28 & 15,38 & 100 & & 28 & 100 \\
\hline \multirow{10}{*}{$\begin{array}{l}\text { CAPES } \\
\text { externe }\end{array}$} & 1995 & 349 & 606 & & & & 196 & & 169 & & & \multirow{10}{*}{$\begin{array}{l}59 \\
34\end{array}$} & & \\
\hline & 1996 & 389 & 671 & & & & 287 & & 223 & & & & & \\
\hline & 1997 & 359 & 693 & & & & 329 & & 266 & & & & & \\
\hline & 1998 & 289 & 723 & 624 & 2,2 & 86,31 & 254 & 40,71 & 209 & 33,49 & 72,32 & & 209 & 72,32 \\
\hline & 1999 & 287 & 749 & 639 & 2,2 & 85,31 & 325 & 50,86 & 287 & 44,9 & 100 & & 287 & 100 \\
\hline & 2000 & 230 & 702 & 586 & 2,5 & 83,48 & 359 & 61,26 & 230 & 39,25 & 100 & & 289 & 125,65 \\
\hline & 2001 & 260 & 725 & 608 & 2,3 & 83,86 & 377 & 62,01 & 260 & 42,76 & 100 & & 294 & 113,08 \\
\hline & 2002 & 303 & 634 & 551 & 1,8 & 86,91 & 385 & 69,87 & 289 & 52,45 & 95,38 & & 289 & 95,38 \\
\hline & 2003 & 280 & 688 & 583 & 2,1 & 84,74 & 383 & 65,69 & 280 & 48,03 & 100 & & 280 & 100 \\
\hline & 2004 & 168 & 724 & 630 & 3,8 & 87,02 & 372 & 59,05 & 168 & 26,67 & 100 & & 168 & 100 \\
\hline \multirow{10}{*}{$\begin{array}{l}\text { Agents } \\
\text { internes }\end{array}$} & 1995 & 42 & & 80 & & & & & 23 & & & & & \\
\hline & 1996 & 35 & & 96 & & & & & 23 & & & & & \\
\hline & 1997 & 26 & & 96 & & & & & 26 & & & & & \\
\hline & 1998 & 23 & 182 & 96 & 4,2 & 52,75 & 34 & 35,42 & 21 & 21,88 & 91,30 & & 21 & 91,30 \\
\hline & 1999 & 20 & 176 & 133 & 6,7 & 75,57 & 33 & 24,81 & 20 & 15,04 & 100 & & 20 & 100 \\
\hline & 2000 & 16 & 180 & 121 & 7,6 & 67,22 & 37 & 30,58 & 16 & 13,22 & 100 & & 16 & 100 \\
\hline & 2001 & 16 & 196 & 138 & 8,6 & 70,41 & 34 & 24,64 & 16 & 11,59 & 100 & & 16 & 100 \\
\hline & 2002 & 16 & 181 & 135 & 8,4 & 74,59 & 36 & 26,67 & 16 & 11,85 & 100 & & 16 & 100 \\
\hline & 2003 & 16 & 200 & 171 & 11 & 85,50 & 36 & 21,05 & 16 & 9,36 & 100 & & 16 & 100 \\
\hline & 2004 & 15 & 181 & 146 & 10 & 80,66 & 35 & 23,97 & 15 & 10,27 & 100 & & 15 & 100 \\
\hline
\end{tabular}

\begin{tabular}{|c|c|c|c|c|c|c|c|c|c|c|c|c|c|c|}
\hline Concours & Année & Postes & Inscrits & Présents & Pré/Poste & $\begin{array}{l}\text { \% Prés } \\
\text { Insc. }\end{array}$ & \begin{tabular}{|l} 
Admisihihs \\
\end{tabular} & $\begin{array}{l}\% \text { admis } \\
\text { Présents }\end{array} \mid$ & Admis & $\begin{array}{l}\text { Admis I } \\
\text { Présents }\end{array}$ & $\begin{array}{c}\text { \% Admis } \\
\text { /Postes }\end{array}$ & LC & Total & $\begin{array}{c}\% \text { Postes } \\
\text { départ }\end{array}$ \\
\hline $\begin{array}{l}\text { CAPES } \\
\text { interne }\end{array}$ & $\begin{array}{l}1995 \\
1996 \\
1997 \\
1998 \\
1999 \\
2000 \\
2001 \\
2002 \\
2003 \\
2004\end{array}$ & $\begin{array}{l}55 \\
60 \\
60 \\
50 \\
65 \\
22 \\
18 \\
20 \\
20 \\
20\end{array}$ & $\begin{array}{c}163 \\
91 \\
173 \\
171 \\
95 \\
108 \\
123\end{array}$ & $\begin{array}{c}114 \\
162 \\
170 \\
122 \\
131 \\
96 \\
132 \\
67 \\
82 \\
95\end{array}$ & $\begin{array}{c}2,4 \\
2 \\
4,4 \\
7,3 \\
3,4 \\
4,1 \\
4,8\end{array}$ & $\begin{array}{c}74,85 \\
143,96 \\
55,49 \\
77,19 \\
70,53 \\
75,93 \\
77,24\end{array}$ & $\begin{array}{l}59 \\
57 \\
46 \\
66 \\
44 \\
46 \\
48\end{array}$ & \begin{tabular}{|c|}
48,36 \\
43,51 \\
47,92 \\
50 \\
65,67 \\
56,10 \\
50,53
\end{tabular} & $\begin{array}{l}19 \\
41 \\
37 \\
44 \\
33 \\
22 \\
18 \\
14 \\
20 \\
20\end{array}$ & $\begin{array}{l}36,07 \\
25,19 \\
22,92 \\
13,64 \\
20,90 \\
24,39 \\
21,05\end{array}$ & $\begin{array}{c}88 \\
50,77 \\
100 \\
100 \\
70 \\
100 \\
100\end{array}$ & 12 & $\begin{array}{l}44 \\
33 \\
22 \\
30 \\
14 \\
20 \\
20\end{array}$ & $\begin{array}{c}88 \\
50,77 \\
100 \\
166,67 \\
70 \\
100 \\
100\end{array}$ \\
\hline $\begin{array}{l}\text { CAPES } \\
\text { réservé }\end{array}$ & \begin{tabular}{l|}
1998 \\
1999 \\
2000 \\
2001 \\
2002 \\
2003 \\
2004
\end{tabular} & $\begin{array}{l}65 \\
\\
71 \\
33 \\
30 \\
10 \\
7\end{array}$ & \begin{tabular}{c|}
170 \\
\\
157 \\
207 \\
145 \\
105 \\
75
\end{tabular} & \begin{tabular}{c|}
127 \\
101 \\
161 \\
74 \\
63 \\
37
\end{tabular} & $\begin{array}{c}2 \\
\\
1,4 \\
4,9 \\
2 \\
6 \\
5\end{array}$ & $\begin{array}{c}74,71 \\
64,33 \\
77,78 \\
51,03 \\
60 \\
49,33\end{array}$ & $\begin{array}{l}70 \\
79\end{array}$ & $\begin{array}{c}55,12 \\
\\
78,22 \\
0 \\
0 \\
0 \\
0\end{array}$ & $\begin{array}{c}55 \\
\\
64 \\
33 \\
25 \\
10 \\
7\end{array}$ & $\begin{array}{l}43,31 \\
\\
63,37 \\
20,50 \\
33,78 \\
15,87 \\
18,92\end{array}$ & $\begin{array}{c}84,62 \\
90,14 \\
100 \\
83,33 \\
100 \\
100\end{array}$ & & $\begin{array}{c}25 \\
10 \\
7\end{array}$ & $\begin{array}{c}83,33 \\
100 \\
100\end{array}$ \\
\hline $\begin{array}{l}\text { Examens } \\
\text { prof. }\end{array}$ & $\begin{array}{l}2001 \\
2002 \\
2003 \\
2004\end{array}$ & & $\begin{array}{c}129 \\
88 \\
82 \\
45\end{array}$ & $\begin{array}{l}115 \\
79 \\
69 \\
41\end{array}$ & & $\begin{array}{l}89,15 \\
89,77 \\
84,15 \\
91,11\end{array}$ & & & $\begin{array}{l}68 \\
54 \\
42 \\
25\end{array}$ & & & & & \\
\hline CAFEP & 1995 & 37 & 49 & & & & 18 & & 14 & & & & & \\
\hline
\end{tabular}




\begin{tabular}{|c|c|c|c|c|c|c|c|c|c|c|c|c|c|c|}
\hline Concours & Année & Postes & Inscrits & Présents & Pré/Poste & $\begin{array}{l}\text { \% Prés } \\
\text { /Insc. }\end{array}$ & Admissibles & \begin{tabular}{l|} 
\% admis \\
/Présents
\end{tabular} & Admis & \begin{tabular}{l|} 
Admis I \\
Présents
\end{tabular} & $\begin{array}{c}\text { \% Admis } \\
\text { /Postes }\end{array}$ & LC & Total & $\begin{array}{c}\text { \% Postes } \\
\text { départ }\end{array}$ \\
\hline CAPES & $\begin{array}{l}1996 \\
1997 \\
1998 \\
1999 \\
2000 \\
200 . \\
2002 \\
2003 \\
2004\end{array}$ & $\begin{array}{l}47 \\
40 \\
34 \\
29 \\
24 \\
20 \\
25 \\
24 \\
15\end{array}$ & $\begin{array}{c}65 \\
81 \\
86 \\
117 \\
99 \\
102 \\
76 \\
90 \\
96\end{array}$ & $\begin{array}{l}72 \\
98 \\
73 \\
77 \\
59 \\
71 \\
69\end{array}$ & $\begin{array}{c}2,1 \\
3,4 \\
3 \\
3,9 \\
2,4 \\
3 \\
5\end{array}$ & $\begin{array}{l}83,72 \\
83,76 \\
73,74 \\
75,49 \\
77,63 \\
78,89 \\
71,88\end{array}$ & $\begin{array}{l}28 \\
21 \\
21 \\
34 \\
29 \\
28 \\
27 \\
38 \\
31\end{array}$ & $\begin{array}{c}29,17 \\
34,69 \\
39,73 \\
36,36 \\
45,76 \\
53,52 \\
44,9\end{array}$ & $\begin{array}{l}15 \\
22 \\
18 \\
29 \\
23 \\
20 \\
21 \\
24 \\
15\end{array}$ & $\begin{array}{c}25 \\
29,59 \\
31,51 \\
25,97 \\
35,59 \\
33,80 \\
21,74\end{array}$ & $\begin{array}{c}52,94 \\
100 \\
95,83 \\
100 \\
84 \\
100 \\
100\end{array}$ & & $\begin{array}{l}18 \\
29 \\
23 \\
20 \\
21 \\
24 \\
15\end{array}$ & $\begin{array}{c}52,94 \\
100 \\
95,83 \\
100 \\
84 \\
100 \\
100\end{array}$ \\
\hline $\begin{array}{l}\text { CAERPA } \\
\text { AG }\end{array}$ & \begin{tabular}{l|}
1998 \\
1999 \\
2000 \\
2001 \\
2002 \\
2003 \\
2004
\end{tabular} & $\begin{array}{l}1 \\
1 \\
1 \\
1 \\
1 \\
1 \\
1\end{array}$ & $\begin{array}{l}18 \\
17 \\
16 \\
26 \\
19 \\
21 \\
23\end{array}$ & \begin{tabular}{c|}
10 \\
12 \\
9 \\
18 \\
14 \\
13 \\
19
\end{tabular} & $\begin{array}{c}10 \\
12 \\
9 \\
18 \\
14 \\
13 \\
19\end{array}$ & $\begin{array}{l}55,56 \\
70,59 \\
56,25 \\
69,23 \\
73,68 \\
61,90 \\
82,61\end{array}$ & $\begin{array}{l}2 \\
2 \\
4 \\
4 \\
3 \\
4 \\
2\end{array}$ & $\begin{array}{c}20 \\
16,67 \\
44,44 \\
22,22 \\
21,43 \\
30,77 \\
10,53\end{array}$ & $\begin{array}{l}1 \\
1 \\
0 \\
1 \\
1 \\
1 \\
1\end{array}$ & $\begin{array}{c}10 \\
8,33 \\
0 \\
5,5 \\
7,14 \\
7,69 \\
5,26\end{array}$ & $\begin{array}{c}100 \\
100 \\
0 \\
100 \\
100 \\
100 \\
100\end{array}$ & & $\begin{array}{l}1 \\
1 \\
0 \\
1 \\
1 \\
1 \\
1\end{array}$ & $\begin{array}{c}100 \\
100 \\
0 \\
100 \\
100 \\
100 \\
100\end{array}$ \\
\hline CAPES & 1995 & 19 & & 73 & & & & & 19 & & & & & \\
\hline CAERPC & $\begin{array}{l}1996 \\
1997 \\
1998 \\
1999 \\
2000 \\
2001 \\
2002 \\
2003 \\
2004\end{array}$ & $\begin{array}{l}30 \\
29 \\
16 \\
35 \\
33 \\
33 \\
43 \\
43 \\
42\end{array}$ & \begin{tabular}{c|}
90 \\
106 \\
115 \\
121 \\
110 \\
99 \\
104
\end{tabular} & $\begin{array}{c}75 \\
94 \\
75 \\
84 \\
94 \\
105 \\
95 \\
83 \\
96\end{array}$ & $\begin{array}{l}4,7 \\
2,4 \\
2,8 \\
3,2 \\
2,2 \\
1,9 \\
2,3\end{array}$ & $\begin{array}{l}83,33 \\
79,25 \\
81,74 \\
86,78 \\
86,36 \\
83,84 \\
92,31\end{array}$ & $\begin{array}{l}42 \\
45 \\
57 \\
55 \\
58 \\
57 \\
69\end{array}$ & $\begin{array}{c}56 \\
53,57 \\
60,64 \\
52,38 \\
61,05 \\
68,67 \\
71,88\end{array}$ & $\begin{array}{l}30 \\
29 \\
16 \\
35 \\
33 \\
33 \\
32 \\
35 \\
38\end{array}$ & $\begin{array}{l}21,33 \\
41,67 \\
35,11 \\
31,43 \\
33,68 \\
42,17 \\
39,58\end{array}$ & $\begin{array}{c}100 \\
100 \\
100 \\
100 \\
74,42 \\
81,40 \\
90,48\end{array}$ & 17 & $\begin{array}{l}16 \\
35 \\
33 \\
50 \\
32 \\
35 \\
38\end{array}$ & $\begin{array}{c}100 \\
100 \\
100 \\
151,52 \\
74,42 \\
81,40 \\
90,48\end{array}$ \\
\hline
\end{tabular}

\begin{tabular}{|c|c|c|c|c|c|c|c|c|c|c|c|c|c|c|}
\hline Concours & Année & Postes & Inscrits & Présents & Pré/Poste & $\begin{array}{l}\text { \% Prés } \\
\text { /Insc. }\end{array}$ & Admissibles & \begin{tabular}{|c|}
$\%$ admis \\
/Présents
\end{tabular} & Admis & $\begin{array}{l}\text { Admis } \\
\text { Présents }\end{array}$ & $\begin{array}{c}\text { \% Admis } \\
\text { /Postes }\end{array}$ & LC & Total & $\begin{array}{c}\text { \% Postes } \\
\text { départ }\end{array}$ \\
\hline $\begin{array}{l}\text { CAPES } \\
\text { spécifique }\end{array}$ & $\begin{array}{l}1995 \\
1996 \\
1997 \\
1998 \\
1999 \\
2000 \\
2001 \\
2002 \\
2003 \\
2004\end{array}$ & $\begin{array}{l}24 \\
28 \\
26 \\
25\end{array}$ & 122 & $\begin{array}{l}64 \\
47 \\
28 \\
61\end{array}$ & 2,4 & 50 & & & $\begin{array}{l}24 \\
28 \\
22 \\
25\end{array}$ & 40,98 & 100 & & & \\
\hline
\end{tabular}

Annexe 2 : Statistiques de tous les concours de recrutement (1995 - 2004)

\section{NOTES}

1. Puis, NAPOLEON fonda la nouvelle Université impériale quelques années plus tard (Loi du 10 mai 1806 et décret du 17 mars 1808).

2. DAURIAC, Lionel, (1847-1923), alors professeur à Montpellier, vint y faire des cours libres d'esthétique musicale.

3. DELAHAYE, Michel ; PISTONE, Danielle. Musique et musicologie dans les Universités françaises, Champion, 1982, p. 17.

4. Dès 1872, dans l'Alsace allemande, Gustav JACOBSTHAL (1845-1912) assura un enseignement d'histoire de la musique à l'Université de Strasbourg.

5. DELAHAYE, Michel ; PISTONE, Danielle. Ibid, p. 31.

6. 21 sur 71 (29\%) en 1982. Cf. DELAHAYE, Michel ; PISTONE, Danielle. Ibid, p. 13. 23 sur 84 Universités (27\%) en 1996. Cf. GANVERT, Gérard. L'enseignement de la musique en France, L'Harmattan, 1999, p. 48.

7. MACHABEY, Armand. La musicologie, PUF, 1962.

8. DELAHAYE, Michel; PISTONE, Danielle. Ibid, p. 12.

9. DELAHAYE, Michel ; PISTONE, Danielle. Ibid, p. 12.

10. Licence, Master, Doctorat.

11. Revue de musicologie (tome 87), 2001, pp. 217-235.

12. "À l'article premier de l'arrêté du 12 septembre 1988 susvisé, les termes : 'Section éducation musicale et chant choral' sont remplacés par les termes : 'Section musique“. Arrêté 7 juillet 2000.

13. MAESTRACCI, Vincent. "L'éducation musicale aujourd'hui : une histoire et des perspectives“, L'éducation musicale de la maternelle à l'Université, Sceren/CRDP Bourgogne, 2003,p.14. 
14. Décret du 7 juin 1991, complété par celui du 4 septembre 1991.

15. BO du 18 juillet 2002

16. Cf. en particulier AUGUGLIARO, Richard. Concours externe de recrutement de professeurs des écoles: Musique: session 2003, SCEREN / CRDP NICE, 2004. Ce recueil rassemble de nombreuses informations : textes officiels, commentaires de l'Inspection générale, différents rapports de jury académiques, une sélection de sujets et un glossaire.

17. BO du 26 mai 2005.

18. http ://www.education.gouv.fr/discours/2002/artssup.htm

19. "Les formations en IUFM“, L'éducation musicale de la maternelle à l'Université, Ibid, pp. 264-265.

20. "Les concours de recrutement et la formation professionnelle“, L'éducation musicale de la maternelle à l'Université, Ibid, pp. 239-245.

21. http ://www.educnet.education.fr/musique/accueil/index.htm

22. La section IALH-Musique fondée en 2000 s'est appuyée pour sa création sur la formation de l'IFUCOME (Institut de formation de l'Université catholique de l'Ouest aux métiers de l'enseignement) existant depuis 1991 en musique pour la formation continue.

23. Les autres options sont les arts plastiques, l'EPS, les langues et cultures régionales, les langues vivantes étrangères et les technologies et ressources éducatives.

24. BO du 13 juin 2002.

25. Sections d'enseignement général et professionnel adapté qui ont pour vocation à accueillir des élèves qui, à l'issue de la scolarité élémentaire, cumulent des retards importants dans les apprentissages scolaires, sans toutefois présenter de retard mental. A à l'issue de la formation en collège, les SEGPA ont pour objectif de permettre d'accéder à une formation professionnelle en lycée professionnel ou en centre de formation d'apprentis.

26. Annexe 1 : Pyramide des âges des professeurs d'éducation musicale.

27. 361837 enseignants dont 347771 titulaires et 14066 non titulaires en 1996-1997. Répartition des professeurs d'éducation musicale par postes occupés: 4766 en collèges, 2 en lycées professionnels, 165 en lycées d'enseignement général et technologique. Total auquel il faut ajouter 1308 enseignants pour le secteur privé. GANVERT, Gérard. L'enseignement de la musique en France, L'Harmattan, 1999, p. 44.

28. Pour le privé : CAERPC (Concours d'accès à l'échelle de rémunération des professeurs certifiés) et CAERPA (Concours d'accès à l'échelle de rémunération des professeurs agrégés).

29. CAPES interne (BO du 20 avril 2000) et agrégation interne (JO du 29 juillet 2000).

30. "Les formations universitaires en musique et musicologie: enseignement et recherche. Quelles perspectives professionnelles?“, L'éducation musicale de la maternelle à l'Université, Ibid, p.236.

31. Annexe 2 : Statistiques de tous les concours de recrutement (1995-2004).

32. Arrêté du 16 avril 1997.

33. Initialement, une épreuve à l'écrit et la seconde à l'oral. Ces modalités furent réformées par la suite avec deux oraux distincts, avant d'être restreintes, à partir de la session 2001, à une seule épreuve orale.

34. Loi $n^{\circ}$ 2001-2 du 3 janvier 2001 parue au JO du 4 janvier 2001.

35. http ://www.omf.paris4.sorbonne.fr/gsem/jpmialaret/ 


\section{RÉSUMÉS}

Disciplines relativement récentes à l'Université, la musique et l'éducation musicale sont dorénavant bien implantées en France. Pierre Gaucher retrace cette évolution qui s'est manifestée par une augmentation importante du nombre d'étudiants et des lieux de formation. Ce texte décrit le cursus universitaire des départements de musique-musicologie, puis des IUFM.

Although music teaching is quite recent at the university it is well established in France. Pierre GAUCHER describes its evolution which was marked by the increasing number of students and of training institutes. This paper examines degree courses in music-musicology departments at the university and at the IUFM.

\section{AUTEUR}

\section{PIERRE GAUCHER}

Maître de conférences, IUFM Montpellier 